\title{
A need for new analytical approach for assessment zearalenone exposure of pigs
}

\author{
Abbreviations: ZEA, Zearalenone; PMTDI, provisional \\ maximum tolerable daily intake.
}

\section{Editorial}

Mycotoxins are secondary metabolites produced by wide variety toxigenic fungi that can occur in all agricultural commodities causing great economic losses at all levels of food and feed production, including crop yields and animal production. ${ }^{1}$ Mycotoxin contaminated diets can reduce performance in ruminants and nonruminants and can have detrimental effects on both finishing animals and reproducing males and females. ${ }^{2}$ Despite that there are over than 500 of mycotoxins described in the literature, only the number of known mycotoxins that pose a measurable health risk to farm animals and humans are covered by EU and world legislation (aflatoxins, ochratoxinA, deoxinivalenol, zearalenone and fumonisins). ${ }^{3}$

In contrast to many other mycotoxins, zearalenone (ZEA) exhibits a very low acute toxicity, but it possesses a powerful estrogenic activity exceeding that of most other naturally occurring non-steroidal estrogens. ${ }^{4}$ Therefore, the presence of ZEA is of the greatest concern for reproducing animals, especially pigs which are the most susceptible species. $^{5}$ This is supported by the fact that JECFA ${ }^{6}$ concluded that the safety of zearalenone could be evaluated on the basis of the dose that had no hormonal effects in pigs. JECFA established a provisional maximum tolerable daily intake (PMTDI) for zearalenone of $0.5 \mu \mathrm{g} /$ $\mathrm{kg}$ of body weight.

Zearalenone is a macrocyclic $\beta$-resorcylic acid lactone produced by several Fusarium species. ${ }^{7}$ ZEA and its derivatives have been detected in many important crops, such as corn, wheat, sorghum, barley, oats, sesame seed, hay and corn silage. ${ }^{8,9}$ Several studies carried out in Europe have reported a high incidence of ZEA in cereals and feeds, most often in Central (Slovakia, Czech Republic), Eastern (Serbia, Romania, Croatia) and Southern (Greece, Italy, Spain, Portugal) parts. ${ }^{10}$ Although in most cases the concentrations of ZEA and other mycotoxins remained below the maximum tolerable levels set by the EU $\left(100 \mu \mathrm{g} / \mathrm{kg}\right.$ for feedingstuffs for piglets and gilts),${ }^{11}$ it is perceivable that long term co-exposure to various mycotoxins will impair animal health and productivity. ${ }^{12,13}$ The presence of ZEA in pigs feed often can have detrimental impact on production and reproduction, leading to great economic losses for producers. According to Visconti ${ }^{14}$ pig producers are affected by increased costs because of higher mortality rates, reproductive failures (abortion), reduced feed efficiency (higher feed costs, lower live weight, infertility syndrome, increased susceptibility to disease), overall quality loss and analytical testing. It was estimated that a 10 or $20 \%$ reduction in farrowing rate combined with a 10 or $20 \%$ reduction in growth (in cases of co-contaminated with zearalenone and deoxinivalenol feed) would result in a 17 to $44 \%$ reduction in profit margins, due to searching for uncontaminated feed, Increased health care and veterinary costs per head, and loss of markets. $^{15}$

There are well established analytical techniques for determination and quantification of ZEA from almost every commodity, mainly
Volume I Issue 4 - 2014

\author{
Georgi Beev \\ Department of Biochemistry, Microbiology and Physics, Trakia \\ University, Bulgaria
}

\begin{abstract}
Correspondence: Georgi Beev, Department of Biochemistry, Microbiology and Physics, Agricultural Faculty, Trakia University, Student Campus, Stara Zagora 6000, Bulgaria, Email gbeev@abv.bg
\end{abstract}

Received: July 30, 2014 | Published: July 31, 2014

based on the GS, GS-MC, HPLC, LC-MC and ELISA for the initial screening. However, the problem with these powerful tools for micotoxicological investigations remains obtaining representative samples. ${ }^{16}$ Furthermore, mycotoxins can be "masked" by certain nutrients making them undetectable. This can be possible explanation for the observed discrepancy between reported data of the prevalence of contamination with ZEA in feeds, and the number of described, proven cases of field outbreaks of zearalenone-toxicoses. ${ }^{17}$ In most cases suspected zearalenone-toxicoses detected by nutritionists and veterinarians remain unreported and the etiological agent responsible for the observed signs is not confirmed when health and production problems improve or disappears following removal of the suspected diets. Sometimes, conclusions could be drawn from herd observations rather than planned experiments, and ZEA is often not measured in the feeds involved. ${ }^{18}$

Under field conditions, ZEA, like other mycotoxins usually occur in concentrations leading to reduced animal performance without causing any obvious clinical symptoms. Moreover, clinically observed symptoms of ZEA exposure could not only be result of the actual (measured) toxin concentration in a given feed, but may also be induced or modulated as the consequence of previous exposure. This is of practical relevance, because the 'historical exposure' of an animal is usually unknown but should be recognized as a possible contributor to disease expression. ${ }^{19}$ For the reasons described above it is difficult to make a link between suspected mycotoxicoses of livestock and the presence of mycotoxins, using standards analytical techniques. Therefore, there is an increasing demand for developing innovative, modern, easy and fast tools for correct diagnostic of zearalenone-toxicoses in pigs.

A possible approach to achieve this goal is so called "omics" technologies and especially transcriptomics that use the ability of living cells to respond to the presence of toxins altering many parameters (including thousands of mRNAs and recently found microRNAs) and leaving specific fingerprints in the form of gene expression, according to their type. The effects on gene expression alterations precede clinical effects. Therefore, mechanisms of action can be detected using transcriptional biomarkers provides information for gene signatures, specific for certain compounds and for mode of action shared by groups of compounds. ${ }^{20}$ One major promise of the transcriptomics is that it could increase our knowledge about toxic 
mechanisms on basis of which the hazard and potentially the risk of a toxic compound can be assessed.

\section{Acknowledgments}

None.

\section{Conflicts of interest}

Author declares that there is no conflict of interest.

\section{References}

1. Beev G, Denev S, Pavlov D. Occurrence and distribution of Fusarium species in wheat grain. Agr Sci Technol. 2011;3(2):165-168.

2. https://www.extension.purdue.edu/extmedia/AS/AS-598-W.pdf

3. Streit E, Schatzmayr G, Tassis P, et al. Current situation of mycotoxin contamination and co-occurrence in animal feed--focus on Europe Toxins (Basel). 2012;4(10):788-809.

4. Metzler M, Pfeiffer E, Hildebrand AA. Zearalenone and its metabolites as endocrine disrupting chemicals. World Mycotoxin Journal. 2010;3(4):385-401.

5. EFSA. Scientific opinion on the risks for public health related to the presence of zearalenone in food. EFSA Journal. 2011;9(6):2197.

6. WHO. Zearalenone. In: Safety Evaluation of Certain Food Additives and Contaminants, WHO Food Additives Series 44, International Programme on Chemical Safety, Prepared by the Fifty-third meeting of the Joint FAO/WHO Expert Committee on Food Additives (JECFA), World Health Organization: Geneva; 2000.

7. Beev G, Denev S, Bakalova D. Zearalenone - producing activity of Fusarium graminearum and Fusarium oxysporum isolated from Bulgarian wheat. Bulg J Agric Sci. 2013;19(2):255-259.

8. Kuiper-Goodman T, Scott PM, Watanabe H. Risk assessment of the mycotoxin zearalenone. Regul Toxicol Pharm. 1987;7(3):253-306.

9. D’Mello JPF, Placinta CM, Macdonald AMC. Fusarium mycotoxins: a review of global implications for animal health, welfare and productivity. Animal Feed Science and Technology. 1999;80(3-4):183-205.
10. Rodrigues I, Naehrer K. A three-year survey on the worldwide occurrence of mycotoxins in feedstuffs and feed. Toxins (Basel). 2012;4(9):663-675.

11. Commission Recommendation. Presence of deoxynivalenol, zearalenone, ochratoxin A, T-2 and HT-2 and fumonisins in products intended for animal feeding. Official Journal of the European Union L. 2006;229:7.

12. Streit E, Naehrer K, Rodrigues I, et al. Mycotoxin occurrence in feed and feed raw materials worldwide: long-term analysis with special focus on Europe and Asia. J Sci Food Agric. 2013;93(12):2892-2899.

13. Stoev SD, Denev SA. Porcine/chicken or human nephropathy as the result of joint mycotoxins interaction. Toxins (Basel). 2013;5(9):1503-1530.

14. Visconti A. Problems associated with Fusarium myco-toxins in cereals. Bulletin of the Institute for Comprehensive Agricultural Sciences. Kinki University: Nara, Japan. 2001;9:39-55.

15. Charmley LL, Trenholm HL, Prelusky DB, et al. Economic losses and decontamination. Nat Toxins. 1995;3(4):199-203.

16. Whitaker TB. Standardisation of mycotoxin sampling procedures: an urgent necessity. Food Control. 2003;14(4):233-237.

17. Pascale M, Visconti A. Overview of detection methods for mycotoxins. In: Leslie JF, Bandyopadhyay R, Visconti A, editors. Mycotoxins. detection methods, management, public health and agricultural trade. CABI Publishing: Wallingford, UK; 2007. p. 171-184.

18. Prodanov-Radulovic JZ, Dosen RD, Stojanov IM, et al. Influence of mycotoxin zearalenone on the swine reproductive failure. $J \mathrm{Nat} \mathrm{Sci}$ Matica Srpska Novi Sad. 2013;124:121-129.

19. Fink-Gremmels J, Malekinejad H. Clinical effects and biochemical mechanisms associated with exposure to the mycoestrogen zearalenone. Anim Feed Sci Technol. 2007;137(3-4):326-341.

20. Pielaat A, Barker GC, Hendriksen P, et al. A foresight study on emerging technologies: state of the art of omics technologies and potential applications in food and feed safety. REPORT 1: Review on the state of art of omics technologies in risk assessment related to food and feed safety. EFSA Supporting Publication, EN-495; 2013. 126 p. 